Lexis Vol. XLV (1) 2021: 163-190

\title{
El peso de la edad en el uso del imperfecto y pluscuamperfecto de subjuntivo $(-r a /-s e)^{*}$
}

\author{
Anahís Samamé Rispa \\ https://orcid.org/0000-0002-7079-3873 \\ Pontificia Universidad Católica del Perú \\ anahis.samamer@pucp.edu.pe
}

\section{RESUMEN}

En este trabajo, se analiza el uso de la variable imperfecto y pluscuamperfecto de subjuntivo -ra/-se en el castellano limeño desde una perspectiva variacionista. A partir de una muestra de hablantes con formación universitaria dividida en tres grupos etarios (jóvenes, adultos y mayores), se propone determinar si -ra está desplazando a -se. Para lo anterior, se analiza si la elección de una u otra variante está condicionada por la edad. Se concluye que el uso de -ra supera al de -se en la muestra estudiada. Asimismo, se comprueba que mientras los jóvenes prefieren más -ra que los adultos y mayores, los últimos usan más -se, lo que confirma que una forma está desplazando a la otra en el castellano limeño.

Palabras clave: variación morfológica, variable, castellano, Lima

\footnotetext{
* Este artículo presenta parte de los resultados de mi trabajo de tesis de maestría, el cual fue financiado por el Programa de Apoyo a la Investigación para Estudiantes de Posgrado (PAIP) 2018 de la PUCP. Se agradecen los comentarios brindados por los profesores de la Maestría y, en especial, los de mi asesor de tesis, Jorge Iván Pérez. Cualquier error recae sobre mi responsabilidad.
} 
The Weight of Age in the Use of the Imperfect and Pluperfect Subjunctive (-ral-se)

\section{Abstract}

This paper focuses on analyzing the use of the imperfect and pluperfect subjunctive - $r a /$-se in the Spanish spoken in Lima from a variational perspective. From a sample of university speakers of three age groups (young, adults and older), it is proposed to determine if $-r a$ is displacing -se, for which it will be analyzed if the election of one or another variant is conditioned by age. It is concluded that the use of -ra exceeds that of $-s e$ in the sample studied. Likewise, it is found that while young people prefer more $-r a$ than adults and older people, the later ones use more -se, which confirms that one form is displacing the other in the Spanish of Lima.

Keywords: morphological variation, variable, Spanish, Lima

\section{Introducción}

Con la introducción de los trabajos de Labov (1972) sobre el inglés americano en los años sesenta, surge un nuevo modelo de análisis para el estudio del lenguaje: la sociolingüística variacionista. Esta disciplina propone que es posible estudiar la variación de una lengua en un contexto real: se trata de analizar el lenguaje en uso para probar que la variación no es aleatoria ni casual, sino sistemática. Aunque, en sus inicios, la mayor parte de los trabajos labovianos o variacionistas se aplicaron a fenómenos fonológicos, no pasaron muchos años para que se tomen en cuenta casos de variación sintáctica: entre los primeros trabajos se encuentran los de Weiner y Labov (1983), y Sankoff y Thibault (1980). En el habla hispana, no son pocos los autores que han tomado en cuenta la variación de tipo morfológico o sintáctico, como la alternancia entre formas verbales de subjuntivo/condicional/indicativo en prótasis de condicionales (Lavandera 1979), el dequeísmo (Serrano 1998), el orden VO en lugar de OV en castellano andino (Klee, Tight y Caravedo 2011), etc. A pesar de que no se ha dejado de problematizar la aplicación del concepto de variable sociolingüística a casos de alternancia morfológica o sin- 
táctica, las investigaciones en estos últimos campos no son escasas, como ya se vio. En este trabajo, se propone estudiar un tipo de variación morfológica del español, el uso de las formas de imperfecto y pluscuamperfecto de subjuntivo (-ra/-se), desde el campo de la sociolingüística variacionista. Interesa investigar si la variante -ra está desplazando a la variante -se en la muestra estudiada, para lo cual se analizará si la elección de una u otra variante está condicionada por la variable social edad ${ }^{1}$. Este tema ha sido estudiado de forma detallada —en su mayoría, desde una perspectiva sociolingüísticaen otros países de Latinoamérica, así como en España (Blas y Porcar 1994, Kempas 2011, Rojo y Vásquez 2014, Navarro 1990, Asratián 2007), pero no en el Perú. En ese sentido, resulta relevante la presente investigación, ya que permitirá cubrir un vacío en los estudios sociolingüísticos del español. Asimismo, contribuirá a aumentar el bagaje de estudios sociolingüísticos de corte variacionista en el país, donde aún no se cuenta con una amplia tradición de investigaciones en este campo, sobre todo, en lo que respecta a variación gramatical. A su vez, ayudará a definir el estado de la variable en cuestión en el español limeño a partir de una muestra de hablantes.

En el siguiente apartado, se revisa, de manera breve, la discusión en torno a la aplicación del concepto de variable a casos de variación no fonológica (morfológica o sintáctica, por ejemplo), así como la importancia de estudios en tiempo aparente. Además, se ofrece un panorama sucinto del estado de este fenómeno de variación a través de la revisión de algunos estudios sobre el tema en España y Venezuela. Luego, se explica la metodología seguida para la obtención de los datos: se específica la muestra de hablantes y el tipo de cuestionario utilizado para elicitar las formas de interés. En la cuarta sección, se detalla el análisis de los datos y los resultados obtenidos. Finalmente, se ofrecen las conclusiones a las que el análisis permite llegar.

El trabajo de tesis del que parte este artículo incluye, también, el estudio de otras variables sociales, como la de género, y de variables lingüísticas. En este escrito, solo se presentan los resultados obtenidos para la edad. 


\section{Estado del arte}

En este apartado, se discute, brevemente, la ampliación de los estudios variacionistas a casos de variación que van más allá del plano fonológico. Así, se explica cómo el concepto de espacios de variabilidad de Caravedo $(1991,2014)$ resulta útil para el estudio de formas alternantes que implican significados distintos, como el del presente trabajo. Se hace referencia, además, a la importancia de la variable edad en el estudio del proceso de cambio lingüístico. Por último, se brinda un recuento de los estudios sobre la alternancia -ra/-se llevados a cabo en España y Venezuela.

\subsection{El concepto de variable más allá del plano fonológico}

Como ya se mencionó, el paso del análisis variacionista a casos de variación morfológica o sintáctica ha sido problematizado antes. En 1978, Lavandera escribe un artículo en el que cuestiona la utilidad del concepto de variable sociolingüística, entendida como dos o más maneras de decir lo mismo, para el estudio de casos que van más allá del plano fonológico. Una de las complicaciones que señala la autora reside en el hecho de que los elementos morfológicos o sintácticos portan significado referencial, del que carecen las variables fonológicas analizadas en los inicios del modelo laboviano. Lo anterior plantea la dificultad de mantener el requisito de equivalencia semántica para una variable lingüística, ya que, a diferencia de lo que sucede en fonología, es más complicado demostrar que se está frente a dos formas distintas de decir lo mismo. Para la autora, la solución consistiría en "[...] relajar la condición de que el significado referencial deba ser el mismo para todas las formas alternantes, y sustituirla por una condición de comparabilidad funcional" [traducción propia] (1978: 181).

Al respecto, Labov, en un artículo en respuesta, se pronuncia y, aunque no se enfoca de manera directa en la propuesta de Lavandera de omitir el requisito de equivalencia semántica y reemplazarlo por el de comparabilidad funcional, concuerda con la autora en que las diferencias de significado no se deben dejar de lado en el estudio 
de la variación (Labov 1978: 8). Por su parte, Romaine responde a ambos autores antes mencionados y señala que se necesitaría de una teoría más integral capaz de explicar la variación de distinta clase, aspecto en que el variacionismo de Labov fallaría (1981: 23). Así, Romaine concuerda con Lavandera en que no es adecuado extender el concepto de variable entendida desde el plano fonológico para casos de variación que van más allá de este. Propone que las variantes deben entenderse como formas con una función común, para lo que el concepto de comparabilidad funcional sería crucial (1981: 22).

Aunque la discusión es más amplia y profunda de lo que se presenta arriba ${ }^{2}$, lo que en este trabajo interesa es justificar la alternancia -ral-se como un caso de variación morfológica que se puede estudiar desde el variacionismo. Para esto, se podrían citar a los autores que se han mostrado a favor de la interpretación de las formas -ra y -se como equivalentes semánticamente (Alarcos Llorach 1949, citado en Lamíquiz 1971; Alvar y Pottier 1983; RAE 2009). Lo anterior resultaría por demás simplificador — sobre todo cuando ya se han comprobado diferentes matices de significado entre las formas para el castellano limeño (Samamé 2018b) — y no resolvería en absoluto el problema de fondo. Entonces, una solución viable es trabajar con el concepto de espacios de variabilidad de Caravedo (1991, 2014): "Se trata de un continuum sonoro dentro del cual se hacen posibles ciertas fluctuaciones, que tienen determinados límites [...] [que] pueden o no coincidir con la capacidad discriminativa de significado" (Caravedo 2014: 130-131). Como se puede observar, la definición anterior aplica para la variación de tipo fonológico.

\footnotetext{
2 Una discusión más detallada sobre el concepto de variable y su aplicación al estudio de casos de variación no fonológica se presenta en el trabajo de tesis de la autora (Samamé 2018a) y en Samamé (2018b). Además, son varios los autores que han tratado el problema. Al respecto, puede revisarse el capítulo 5 de Tagliamonte (2006). También, Serrano (2007) presenta un artículo bastante interesante en el que concluye que, en la actualidad, la pregunta sobre si se deben estudiar los casos de variación sintáctica desde una perspectiva variacionista es ya obsoleta cuando su aplicación se encuentra más que justificada.
} 
Para el plano sintáctico y léxico, la autora amplía el concepto y sostiene que "[...] la variación no se expresa necesariamente de modo discreto en entidades claramente delimitadas que alternan entre ellas, sino que puede ondular entre zonas conceptuales distintas, las cuales se acercan y se intersectan en determinados contextos" (Caravedo 2014: 159). Al tratarse de conceptos diferentes que "comparten" ciertos espacios o se "intersectan", y se actualizan en una misma forma, queda superado el principio de equivalencia semántica. Al plantear este estudio un caso de variación morfológica de dos formas diferentes que coaparecen en diversos contextos (-ra y -se), se propone aplicar el concepto anterior para el análisis de este fenómeno. De esta manera, lo que justificaría estudiar a -ra $\mathrm{y}$-se como formas de un mismo continuum es que compartirían un mismo espacio de variabilidad. En otras palabras, se encuentran en un mismo continuum que posibilita su coaparición en los mismos contextos. De lo anterior, se desprende que se trata de un caso de variación posible de estudiar con la metodología variacionista.

2.2. La edad y el cambio lingüístico: tiempo aparente y tiempo real

Para la sociolingüística, el estudio de la edad ha sido importante. Se trata de una variable que permite aproximarse al proceso de cambio lingüístico desde una perspectiva distinta a como había sido estudiado desde la lingüística histórica. Labov plantea el estudio del cambio a partir de una doctrina uniformista: se parte de la idea de que los mecanismos de cambios que operaron en el pasado son los mismos que operan en el presente, los cuales se pueden observar a partir de estudios lingüísticos sincrónicos (1972: 161). Para el autor, es posible realizar observaciones que arrojen datos relevantes para el estudio del cambio a partir de dos generaciones sucesivas de hablantes (1972: 163). Así, el tiempo aparente, aquel representado por los actuales hablantes de una lengua divididos en distintos grupos etarios, se convierte en la posibilidad antes obviada de estudiar el cambio en curso. De lo anterior, se entiende que el cambio es un proceso lingüístico que implica la variación en un estado sincrónico de la lengua, de ahí la importancia del estudio de esta 
última para entenderlo, aunque no se pueda predecir con certeza qué dirección tome la variación de algún elemento de la lengua en un momento determinado, es decir, si se concretará el cambio o no.

En esta investigación, se plantea la variable edad como importante para entender el caso de variación en estudio. Esta variable será útil para describir cómo las pautas de uso del imperfecto y pluscuamperfecto de subjuntivo han cambiado a partir de tres generaciones de hablantes. Lo anterior permitirá comprobar cómo una forma $(-s e)$ está siendo relegada por otra $(-r a)$ en el castellano limeño. Así, el estudio del factor edad brindará luces sobre un posible cambio en curso a partir de la distribución de las formas -ral-se en cada grupo etario. Para dar cuenta de lo anterior, será importante recurrir al concepto de hipercorrección propuesto por Labov para explicar la distribución de la variable (r-1) en su estudio del inglés de los almacenes de Nueva York (1972). Se verá que el prestigio es un concepto importante para explicar la distribución de las formas. De esta manera, se comprobará la utilidad de los estudios en tiempo aparente, es decir, aquellos con datos sincrónicos de la lengua, para entender un poco más sobre los mecanismos involucrados en el proceso de cambio lingüístico.

\subsection{Antecedentes}

Diversos son los estudios que se han referido a la frecuencia de uso de las variantes -ra y -se del castellano en diferentes comunidades de habla, sobre todo en España. En el contexto latinoamericano también se encuentran varios trabajos. En el Perú, sin embargo, solo se han identificado dos estudios que dan cuenta de este fenómeno: uno pertenece a la ciudad de Chincha (Cuba 1996) y el otro, a la de Lima (DeMello 1993). Ambos dedican un espacio a comparar las frecuencias de las variantes de imperfecto y pluscuamperfecto de subjuntivo. En este último estudio, se observa la predominancia de -ra sobre -se. En el primero, en cambio, la autora refiere al hecho de que, en el castellano chinchano, los hablantes optan por -se la mayoría de las veces. Estos trabajos no se proponen estudiar el fenómeno a profundidad desde una perspectiva variacionista, como 
sí sucede en otros países. A continuación, se explican brevemente solo algunos de los estudios realizados en otros contextos, específicamente en España y Venezuela, los cuales centran su objetivo en estudiar este fenómeno de variación desde una perspectiva social.

En cuanto a la frecuencia de uso de las variantes, en España, todos los estudios consultados a los que se hace referencia en el presente artículo llegan a la conclusión de la preferencia de la variante -ra en detrimento de -se (Blas y Porcar 1994, Kempas 2011, Rojo y Vásquez 2014). Respecto a la variable edad como un factor influyente en la elección de una u otra forma, en el trabajo de Blas y Porcar (1994), esta se mostró significativa. Los datos permiten observar que mientras más se avanza en los grupos de edad, menor es el empleo de -se: "[...] entre adultos y ancianos esta variante se emplea muy escasamente” (1994: 87). Los autores relacionan este hecho con la instrucción que reciben los jóvenes en su etapa escolar, ya que serían más conscientes de la intercambiabilidad de las formas que los más adultos.

En cambio, para Rojo y Vásquez (2014), esta variable no se mostró como significativa de forma tan clara. Así, las frecuencias obtenidas mostraron que son las mujeres de los grupos de más edad las que presentan mayor uso de -ra, mientras que los hombres del grupo más joven y de mayor edad utilizan más la forma -se. Sin embargo, más adelante, los autores tienen en cuenta la desviación estándar de los datos para su análisis debido a la gran variabilidad de estos: "[...] el análisis individualizado muestra que 9 de las 18 encuestas no presentan ningún caso de -se y otras dos muestran porcentajes de -ra superiores al 90\%. De las siete restantes, tres tienen porcentajes de -se superiores al 60\% y las demás se sitúan en una zona intermedia” (2014: 253). De este modo, después de tomar en cuenta la desviación estándar, se observa que no hay diferencias significativas entre hombres y mujeres ni en los grupos de edad en cuanto a los usos de -ra y -se, aunque los autores resaltan el hecho de las fuertes diferencias a nivel individual de estos usos.

Por su parte, los datos recogidos por Kempas (2011) no permiten asociar la edad del informante con una variación estadísticamente 
significativa; sin embargo, el investigador resalta el hecho de que los informantes de 25 a 35 años prefieran cantara en mayor medida que los menores de 25 años, lo que podría significar que la frecuencia de uso de cantase estaría en aumento en la generación joven.

En lo que respecta a los estudios de Venezuela, estos pertenecen a las ciudades de Valencia y Caracas. En ambos lugares, los datos demuestran que los usos de -ra sobrepasan a los de-se: mientras que Navarro (1990) indica que, de las 952 formas obtenidas de -ra/-se, la realización en -se solo alcanzó el 9\%, Asratián (2007) demuestra que, en su corpus, el uso de -se (6\%) es reducido en relación con el empleo de -ra (94\%).

Estos autores también se refieren a la influencia que podría tener la edad en la selección de una de las variantes. Así, Asratián señala que el grupo generacional que promueve la aparición de -se es el de 30 a 45 años (peso=0,635), es decir, el grupo de edad media. La autora explica el hecho de que este grupo en particular haya tenido resultados que favorecen la aparición de -se siguiendo a Silva-Corvalán, quien sostiene que las diferencias de uso de una variable lingüística por grupos etarios se deben, con frecuencia, no al factor edad en sí, sino a factores relacionados, como la percepción que el hablante tiene de las ventajas de usar los rasgos de mayor prestigio. Así, al ser los hablantes de edad media más activos económica y laboralmente, los resultados podrían deberse más a una cuestión de mercado lingüístico - por el que los hablantes de ciertas profesiones tienden a ajustarse a modelos lingüísticos prestigiosos (Moreno 1998: 50, 60 y 351, citado en Asratián 2007: 32) — que a la edad en sí. En otras palabras, los hablantes de estas edades están bajo presiones sociales, las cuales se reflejan en su conducta lingüística al tratar de usar las formas consideradas más prestigiosas, como ocurre con -se. Asimismo, Navarro (1990) encuentra en la edad un factor explicativo para el fenómeno. Los hablantes jóvenes muestran una frecuencia reducida de - se $(2,7 \%)$, la cual aumenta según la edad: 9,7\% para el segundo grupo y $16 \%$ para el grupo de más edad.

Como ya se detalló, el objetivo de este trabajo es, por un lado, comprobar el uso mayoritario de -ra en lugar de -se en la muestra 
seleccionada, resultado que se muestra regular en casi todas las variedades del español que han sido estudiadas. Por otro lado, en cuanto a la variable edad, aunque los resultados de los estudios de España no parecen estar dirigidos en una sola dirección, los dos estudios de Venezuela señalados en los párrafos anteriores serán considerados de relevancia para conocer si el uso de una u otra variante está condicionado por el factor edad. A partir de estos trabajos, se intenta comprobar que la edad es una variable explicativa para la preferencia de una variante sobre otra en el uso del imperfecto y pluscuamperfecto de subjuntivo en el castellano limeño.

\section{Metodología}

\subsection{Población y muestra}

La población objetivo está conformada por todos los hablantes nativos de español nacidos en la ciudad de Lima que se encuentren cursando o que hayan cursado estudios universitarios. Según los datos de Censos Nacionales 2007: XI de Población y VI de Vivienda del INEI, en Lima, habría un total de 843028 personas hablantes de castellano como lengua materna que han terminado los estudios superiores universitarios y 509266 con estudios incompletos. De esta cantidad de personas, no se maneja cuántas de ellas han nacido en Lima - es común que personas de otras ciudades lleguen a la capital para estudiar en la universidad-y cuántas aún se encuentran cursando los estudios de pregrado. Es decir, no se cuenta con el número exacto de personas que son parte de la población de este estudio. Aun así, de los datos, se desprende que el número aproximado sería bastante elevado.

Debido a la cantidad de individuos que conforman la población de esta investigación, la muestra no es representativa ni probabilística. Esto quiere decir que no todos los sujetos del universo han tenido la misma probabilidad de ser elegidos, ya que la selección de la muestra no se realizó a partir de métodos estadísticos (Hernández, Fernández y Baptista 2010: 176). Es así que el instrumento 
de recolección de datos se aplicó solo a aquellas personas a las que la investigadora tuvo acceso y aceptaron ser entrevistadas. El número total de la muestra es de 54 sujetos, y su clasificación por edad y género fue la siguiente: la mitad de los encuestados fueron mujeres (27) y la otra mitad, hombres (27). Además, se distribuyó la muestra en tres grupos de edad: jóvenes, adultos y mayores. Los jóvenes conforman el primer rango. Se trata de 18 hablantes de 20 a 34 años (9 mujeres y 9 hombres). El total de hablantes adultos que pertenecen al segundo rango es 18 e incluye a personas de 35 a 50 años ( 9 mujeres y 9 hombres). Por último, el tercer rango es el grupo de mayores y está conformado por 18 colaboradores de 51 a más años de edad ( 9 mujeres y 9 hombres). Todos los entrevistados cumplen con las características de haber nacido en el departamento de Lima, tener al castellano como lengua materna, y cursar o haber cursado estudios superiores universitarios.

\subsection{Instrumento de recolección de datos}

El instrumento de recolección de datos (IRD) consta de un cuestionario anónimo que contiene cuatro partes. Cada parte del cuestionario buscaba elicitar datos específicos. La primera, por ejemplo, recoge los datos personales del colaborador para clasificar al hablante por edad. En la segunda parte, se les pide a los encuestados ver un video en donde se muestran distintos casos con la misma temática: un padre que ayuda a sus hijos o evita que les ocurra un accidente. Luego, se les indica que, a partir de imágenes de diferentes escenas del video, imaginen situaciones distintas o contrarias a las que observaron y completen con formas verbales los espacios en blanco de preguntas que hacen referencia a la situación de la imagen, así como de las respuestas a esas preguntas. En este segundo apartado del IRD, hay un total de 20 oraciones condicionales (10 hacen referencia a las preguntas; y 10, a las respuestas). La imagen 1 ejemplifica esta segunda parte del IRD. 


\section{Imagen $\mathrm{N}^{\circ}$. Parte II del IRD}

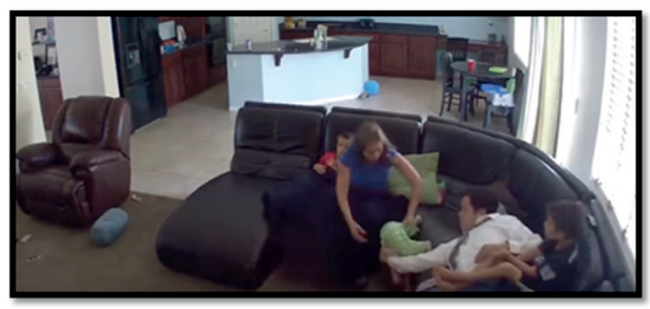

1. ¿Qué habría pasado si el bebé, de todas maneras, se caido ?

El niño se golpeado la

La tercera parte del cuestionario contiene cinco imágenes acompañadas de una breve historia. Según esta, se les pide a los colaboradores completar oraciones que hacen referencia a lo que los personajes pueden estar diciendo o pensando. Esta parte del cuestionario contiene 8 oraciones con espacios en blanco para ser completados con las formas verbales de interés, como se muestra en la imagen 2. La última parte del cuestionario es un texto para completar adaptado del estudio de Williams (1982) para el español de Navarra, España. En este apartado, se incluyen 13 oraciones en las que necesariamente deberían aparecer las formas en subjuntivo y 7 oraciones distractoras para completar con otras formas verbales.

\section{Imagen $\mathrm{N}^{\circ}$ 2. Parte III del IRD}

2. Ella es Samantha. El día de hoy tenía planeado ir al parque con sus amiguitos del colegio para jugar, pero su mamá le dijo que era imposible porque, repentinamente, había comenzado a llover. Ahora Samantha está triste. ¿Qué crees que está pensando?

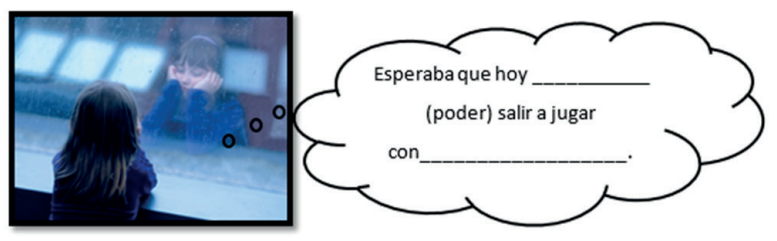




\section{Análisis y resultados}

En este apartado, se presentan el análisis y los resultados de los datos. Estos fueron procesados con el programa estadístico SPSS (versión 20), el cual permite elaborar tablas de frecuencias (absolutas y relativas) y estudiar el comportamiento de la variable dependiente en relación con la independiente a través de correlaciones. Primero, se realiza el conteo general de las variantes -ra y -se. Luego, se ofrecen los datos del número de apariciones de estas formas por cada grupo de edad. Finalmente, se analizan los datos anteriores para comprobar la correlación estadística.

\subsection{Conteo total de datos ${ }^{33}$}

A continuación, se pueden observar los datos referidos al conteo total de las formas obtenidas en este estudio: se presenta el número de veces que cada una de estas aparece, así como los porcentajes correspondientes.

Tabla $\mathrm{N}^{\circ} 1$. Conteo total de los datos

\begin{tabular}{lcc}
\hline & Frecuencia & Porcentaje \\
\hline -ra imperfecto & 646 & 40,6 \\
-se imperfecto & 102 & 6,4 \\
-ra pluscuamperfecto & 620 & 39,0 \\
-se pluscuamperfecto & 222 & 14,0 \\
Total & 1590 & 100,0 \\
\hline
\end{tabular}

3 El total de formas recolectadas en la muestra estudiada a través del IRD fue de 2214. De estas formas, solo 1590 correspondían a las formas de imperfecto y pluscuamperfecto de subjuntivo y el resto, a formas del presente de subjuntivo, condicional, indicativo y otros. En el trabajo de tesis del que parte este artículo, se ofrecen, de manera detallada, las frecuencias y porcentajes obtenidos para cada uno de los elementos verbales recolectados. Por razones de espacio, en el presente artículo, solo se consideran las variantes de -ra y -se para el análisis. 


\section{Gráfico $\mathrm{N}^{\circ}$. Conteo simplificado del total de los datos}

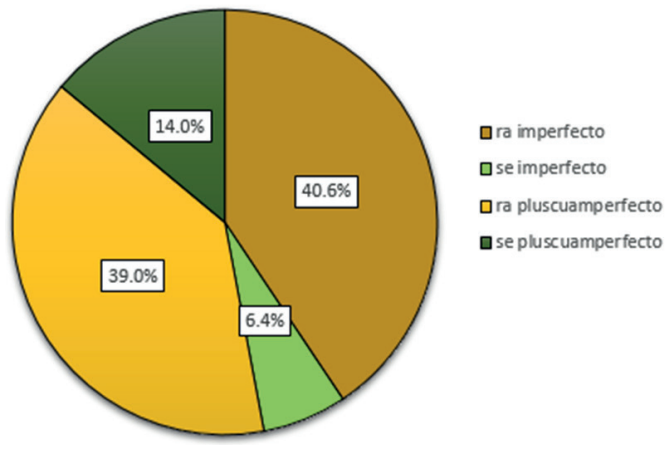

Como se puede observar en la tabla $N^{\circ} 1$ y el gráfico $N^{\circ} 1$, el total de formas que se obtuvo para la variable imperfecto y pluscuamperfecto de subjuntivo a partir de la recolección de los datos fue de 1590. La distribución de las formas ocurrió de la siguiente manera: 1266 veces apareció la forma -ra (79,6\%), con 646 formas de imperfecto $(40,6 \%)$ y 620 apariciones de pluscuamperfecto $(39 \%)$, mientras que la variante -se tuvo 324 apariciones $(20,4 \%)$, de las cuales 102 son de imperfecto $(6,4 \%)$ y 222 son de pluscuamperfecto $(14 \%)$.

Así, casi el $80 \%$ de las ocurrencias pertenecen a la variante -ra, mientras que -se solo obtiene el 20,4\% de apariciones. Estos datos confirman el hecho, mencionado en la amplia bibliografía sobre el tema, de que -ra es la forma más usada de la variable de imperfecto y pluscuamperfecto de subjuntivo. De esta manera, se comprueba que, para el castellano limeño, el patrón de uso de estas variantes es el mismo que para la mayoría de variedades del español: mayor uso de -ra en detrimento de -se.

\subsection{Conteos de la variable edad}

En este apartado, se incluye el conteo de las formas obtenidas por cada grupo o rango de edad: jóvenes (menores de 35 años), adultos (de 35 a 50 años) y mayores (de 51 años a más). A continuación, se muestran los resultados obtenidos para cada uno de ellos. 
4.2.1. Grupo de jóvenes

Tabla $\mathrm{N}^{\circ}$ 2. Conteo del grupo de jóvenes

\begin{tabular}{lcc}
\hline & Frecuencia & Porcentaje \\
\hline -ra imperfecto & 250 & 45,2 \\
-se imperfecto & 25 & 4,5 \\
-ra pluscuamperfecto & 228 & 41,2 \\
-se pluscuamperfecto & 50 & 9,0 \\
Total & 553 & 100,0 \\
\hline
\end{tabular}

En la tabla $\mathrm{N}^{\circ} 2$, se puede observar que la variante -ra tiene un porcentaje mayor al $85 \%$ (45,2\% de imperfecto y $41,2 \%$ de pluscuamperfecto), mientras que las formas en -se presentan 75 apariciones con solo $13,5 \%$ del total (4,5\% de imperfecto y $9 \%$ de pluscuamperfecto). En este grupo de edad, entonces, el porcentaje de las formas en -ra supera en más de 6 veces al de -se; es decir, los jóvenes prefieren usar mucho más -ra que -se.

4.2.2. Grupo de adultos

Tabla $N^{\circ}$ 3. Conteo del grupo de adultos

\begin{tabular}{lcc}
\hline & Frecuencia & Porcentaje \\
\hline -ra imperfecto & 202 & 38,1 \\
-se imperfecto & 48 & 9,1 \\
-ra pluscuamperfecto & 189 & 35,7 \\
-se pluscuamperfecto & 91 & 17,2 \\
Total & 530 & 100,0 \\
\hline
\end{tabular}

En el grupo de hablantes adultos (de 35 a 50 años), como se puede observar en la tabla de frecuencias $\mathrm{N}^{\circ} 3$, -ra obtuvo un total de 391 apariciones con un 73,8\% del total (38,1\% de imperfecto y $35,7 \%$ de pluscuamperfecto). Por su parte, la variante -se aparece 
139 veces, lo que representa el 26,3\% del total (9,1\% de imperfecto y $17,2 \%$ de pluscuamperfecto).

Si se comparan estos datos con los de la tabla anterior, se puede afirmar que el porcentaje de uso de las formas en -ra es más bajo en los adultos que en los jóvenes: existe una diferencia de 11,2 puntos porcentuales menos para los adultos. En cambio, las veces que aparece la variante -se aumentan en este grupo de hablantes; es decir, los hablantes adultos usan más la forma -se que los jóvenes.

4.2.3. Grupo de mayores

Tabla $\mathrm{N}^{\circ}$. Conteo del grupo de mayores

\begin{tabular}{lcc}
\hline & Frecuencia & Porcentaje \\
\hline -ra imperfecto & 194 & 38,3 \\
-se imperfecto & 29 & 5,7 \\
-ra pluscuamperfecto & 203 & 40,0 \\
-se pluscuamperfecto & 81 & 16,0 \\
Total & 507 & 100,0 \\
\hline
\end{tabular}

Respecto al grupo de hablantes mayores (de más de 50 años), se puede observar que la distribución de las formas de imperfecto y pluscuamperfecto de subjuntivo es como sigue: $78,3 \%$ de $-r a$ (38,3\% de imperfecto y $40 \%$ de pluscuamperfecto) y $21,7 \%$ de - se (5,7\% de imperfecto y $16 \%$ de pluscuamperfecto).

Si se tienen en cuenta los datos del grupo de adultos, la distribución de las formas de imperfecto y pluscuamperfecto de subjuntivo no varía de forma relevante con respecto a estos últimos. Por ejemplo, los porcentajes de la forma -ra están por encima del $70 \%$ en ambos grupos y los de -se, por encima del 20\%. En donde se aprecia una variación importante es entre los dos últimos grupos y el primero, el de jóvenes. A continuación, se ofrece un gráfico que agrupa las formas -ra y -se obtenidas sin diferenciar entre imperfecto y pluscuamperfecto con el fin de lograr una comparación más clara. 
Gráfico $\mathrm{N}^{\circ}$ 2. Comparación del conteo de la variable edad
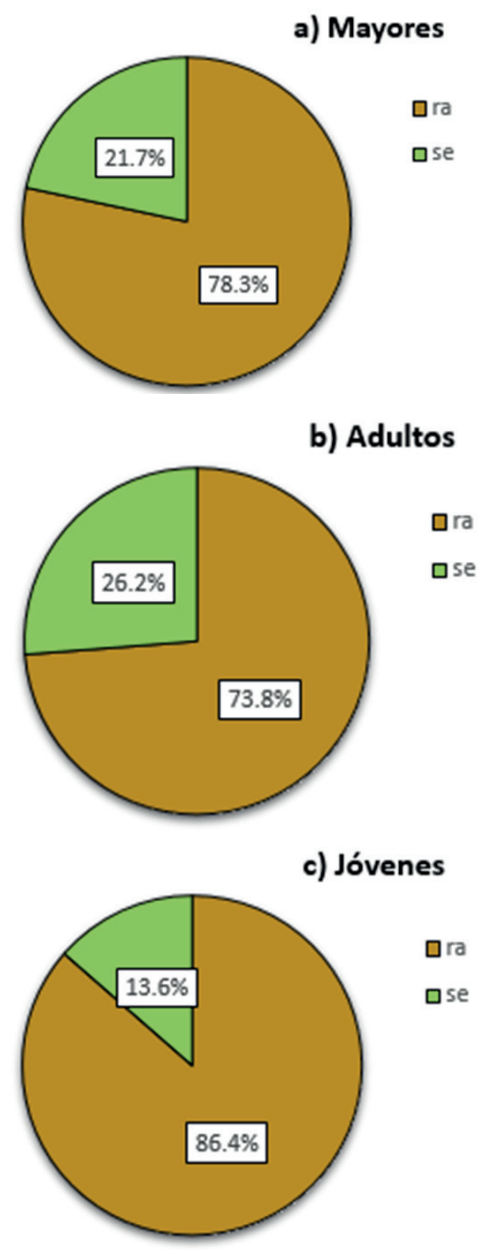

A través de estos gráficos, se puede verificar que la tendencia es que el uso de las formas en -se disminuya en el grupo de hablantes jóvenes respecto del grupo de adultos y mayores, aunque también se observa una ligera disminución del porcentaje de -se en los hablantes mayores en comparación con los adultos. Mientras que los jóvenes solo usaron el 13,6\% de las veces -se, los adultos y los mayores lo 
hicieron el 26,2\% y el 21,7\%, respectivamente. Esto quiere decir que, de los tres grupos de hablantes, son los jóvenes quienes menos usan la variante -se, lo cual comprueba que esta es la forma que se está perdiendo en la actualidad. Además, se muestra que el porcentaje de -ra aumenta en el grupo de jóvenes con respecto a los dos grupos de hablantes de mayor edad: los jóvenes usan 8,1 puntos porcentuales más -ra que los mayores y 12,6 puntos porcentuales más que los adultos. Todo lo anterior se puede observar de forma más clara en el gráfico $\mathrm{N}^{\circ} 3$, que agrupa a los adultos y mayores con $75,8 \%$ de -ra y $24,2 \%$ de -se, por un lado, y a los jóvenes con $86,4 \%$ de - ra y $13,6 \%$ de - se, por otro.

Gráfico $\mathrm{N}^{\circ}$ 3. Comparación del conteo entre dos grupos de edad: adultos y mayores, $\mathrm{y}$ jóvenes

a) Adultos y mayores
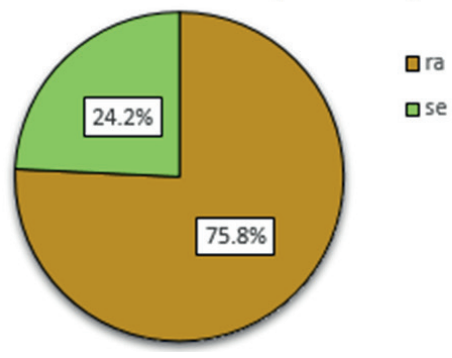

b) Jóvenes

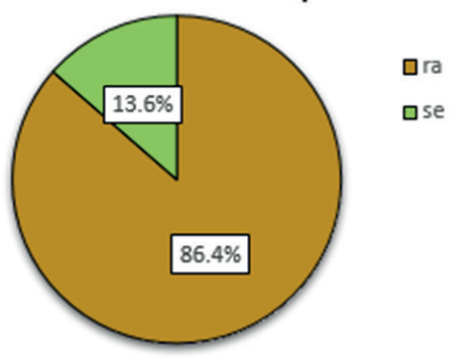

Quedaría por explicar por qué los hablantes adultos usan más la forma -se que los hablantes mayores si esta es la forma que se está perdiendo. Una respuesta plausible se puede encontrar en el concepto de hipercorrección utilizado por Labov (1972) en su estudio del uso de $r$ en Nueva York para explicar por qué la clase media baja muestra más usos de las formas prestigiosas que los hablantes de la clase alta: este concepto está referido al “[...] hecho de que los hablantes de clase media baja superan en sus realizaciones de las formas consideradas como correctas y adecuadas a los estilos formales al grupo de mayor estatus" [traducción propia] (1972: 126). 
El fenómeno de hipercorrección puede ser útil si se toma en cuenta que la variante del imperfecto y pluscuamperfecto de subjuntivo considerada más prestigiosa es -se. Los resultados obtenidos por Valeš (2006) al respecto para el castellano de Granada son bastante determinantes: en su estudio sobre la forma de mayor prestigio, concluye que es -se la variante más culta, prestigiosa y adecuada a los estilos formales. Por su parte, para Latinoamérica, "Las variantes en -se se consideran demasiado elevadas, o incluso afectadas, en la lengua oral de algunos países americanos. [...] algunos hablantes de nivel sociocultural medio o bajo tienden a usarlas en contextos formales porque les atribuyen mayor prestigio" (RAE 2009: 1803). Lo anterior se comprobó con las entrevistas realizadas. Siempre que se tuvo la oportunidad, después de aplicar el IRD, se les consultó a los hablantes por las diferencias que percibían entre una y otra forma. La respuesta general que se obtuvo es la asociación de -se con estilos más formales, cuidados o cultos. En cambio, la forma en -ra se relacionaba con contextos más orales y descuidados. Asimismo, en conversaciones con distintos docentes universitarios que manejan una variedad del español utilizada en el ámbito académico, se obtuvo la misma respuesta: asociación de -se con formas más cultas y cuidadas.

Se propone, entonces, extender la aplicación del concepto de hipercorrección al caso que se plantea en el presente estudio. Como se sabe, los hablantes más activos laboralmente son los que se encuentran entre las edades de 35 a 50 años, es decir, los adultos. Los hablantes entrevistados entre esas edades, al tener todos estudios universitarios y desempeñarse en diferentes ámbitos profesionales, buscan usar formas que les permitan posicionarse como hablantes de la variedad más prestigiosa del castellano. Una forma de hacerlo sería utilizando la variante -se. La mayoría de hablantes mayores, en cambio, eran jubilados o ya no ejercían su profesión, por lo que no presentaban la presión de cuidar sus formas de habla. Además, es conocida en la literatura la idea de que los hablantes con mayor seguridad lingüística son los mayores, por lo que no suelen modificar sus usos ante la dicotomía +prestigio/-prestigio. Por ejemplo, 
Moreno cita a Chambers, quien propone tres etapas formativas para la adquisición del sociolecto ("infancia”, “adolescencia” y "edad adulta joven") y señala que "Después de esa tercera etapa, se supone que los hablantes estabilizan sus sociolectos” (2009: 50). En conclusión, los adultos se hipercorrigen y usan más la variante -se que los mayores, porque esta es la forma de mayor prestigio.

Es importante notar que Labov introduce el concepto de hipercorrección para explicar un cambio desde arriba promovido por los hablantes más jóvenes de clases altas. En la presente sección, en cambio, no se intenta explicar la promoción de una forma usada por los más jóvenes, sino, más bien, el mayor uso de una forma por hablantes adultos en comparación con hablantes mayores. Mientras Labov usa la noción de hipercorrección para explicar el aumento del uso de formas más prestigiosas en hablantes de clase media en comparación con los de clase alta, en este trabajo, se usa para explicar el mayor uso de una forma en hablantes adultos en vez que en mayores con el fin de lograr mayor prestigio. Queda demostrada, así, la importancia de los factores sociales, como la edad o el prestigio asociado a las formas, para entender el camino de un cambio lingüístico.

\subsection{Correlación con la variable edad}

Tabla $N^{\circ}$ 5. Tabulación cruzada: edad y variable dependiente

\begin{tabular}{lccccc}
\hline & & \multicolumn{3}{c}{ Edad } & Total \\
\cline { 3 - 5 } 20 a 34 & 35 a 50 & 51 a más & \\
\hline formas & $-r a$ & 478 & 391 & 397 & 1266 \\
& $-s e$ & 75 & 139 & 110 & 324 \\
Total & 553 & 530 & 507 & 1590 \\
\hline
\end{tabular}

Para conocer si existe relación estadísticamente significativa entre la edad y la variable imperfecto y pluscuamperfecto, se aplicó la técnica de Chi-cuadrado a la muestra teniendo en cuenta el conteo de -ra y -se, tanto imperfecto como pluscuamperfecto, para 
cada grupo de edad. En la tabla $\mathrm{N}^{\circ} 5$, se puede observar el número total de formas obtenidas por los jóvenes (20 a 34), adultos ( 35 a 50) y mayores (50 a más). Para probar la relación, se manejan las dos siguientes hipótesis.

$\mathrm{H}_{0}$ : Las formas de imperfecto y pluscuamperfecto de subjuntivo, y la edad no se relacionan.

$\mathrm{H}_{\mathrm{i}}$ : Las formas de imperfecto y pluscuamperfecto de subjuntivo, y la edad sí se relacionan.

Tabla N6. Prueba de Chi-cuadrado para edad

\begin{tabular}{lccc}
\hline & Valor & Gl & $\begin{array}{c}\text { Sig. asintótica (2 } \\
\text { caras) }\end{array}$ \\
\hline Chi-cuadrado de Pearson & $27,549^{\mathrm{a}}$ & 2 &, 000 \\
Razón de verosimilitud & 28,464 & 2 &, 000 \\
N de casos válidos & 1590 & & \\
\hline
\end{tabular}

Nivel de significación: 0.01

Debido a que el Sig. asintótica de la tabla $\mathrm{N}^{\circ} 20$ es menor al nivel de significación $(0,00<0,01)$, se rechaza la hipótesis nula $\left(\mathrm{H}_{0}\right)$ y la hipótesis de investigación $\left(\mathrm{H}_{\mathrm{i}}\right)$ queda probada. De esta manera, se concluye, con un nivel de significación del 1\%, que las formas de imperfecto y pluscuamperfecto, y la edad están relacionadas estadísticamente. Es decir, se comprueba la hipótesis de asociación para estas variables. Para conocer cómo es que se asocian, se utiliza la prueba de correspondencia simple, cuyos resultados se muestran en la tabla $\mathrm{N}^{\circ} 7$. 
Tabla $N^{\circ}$. Prueba de correspondencia simple para edad y la variable dependiente

\begin{tabular}{|c|c|c|c|c|c|c|}
\hline \multirow[b]{3}{*}{ Forma } & \multirow[b]{3}{*}{ Masa } & \multirow{3}{*}{\begin{tabular}{|c}
$\begin{array}{c}\text { Puntuación en } \\
\text { dimensión }\end{array}$ \\
\\
1 \\
\end{tabular}} & \multirow[b]{3}{*}{ Inercia } & \multicolumn{3}{|c|}{ Contribución } \\
\hline & & & & \multirow{2}{*}{$\begin{array}{c}\text { Del punto en } \\
\text { la inercia de } \\
\text { dimensión }\end{array}$} & \multicolumn{2}{|c|}{\begin{tabular}{|c|}
$\begin{array}{c}\text { De la dimensión } \\
\text { en la inercia del } \\
\text { punto }\end{array}$ \\
\end{tabular}} \\
\hline & & & & & 1 & Total \\
\hline $\mathrm{Ra}$ & ,796 &,- 184 &, 004 & ,204 & 1,000 & 1,000 \\
\hline $\mathrm{Se}$ & 204 & ,717 &, 014 & ,796 & 1,000 & 1,000 \\
\hline $\begin{array}{l}\text { Total } \\
\text { activo }\end{array}$ & 1,000 & &, 017 & 1,000 & & \\
\hline
\end{tabular}

\begin{tabular}{|c|c|c|c|c|c|c|}
\hline \multirow[b]{3}{*}{ Edad } & \multirow[b]{3}{*}{ Masa } & \multirow{3}{*}{$\begin{array}{c}\text { Puntuación en } \\
\text { dimensión } \\
\\
1 \\
\end{array}$} & \multirow[b]{3}{*}{ Inercia } & \multicolumn{3}{|c|}{ Contribución } \\
\hline & & & & \multirow{2}{*}{ 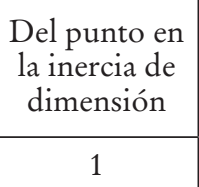 } & \multicolumn{2}{|c|}{$\begin{array}{l}\text { De la dimensión } \\
\text { en la inercia del } \\
\text { punto }\end{array}$} \\
\hline & & & & & 1 & Total \\
\hline 20 a 34 & ,348 &,- 466 & 010 & ,575 & 1,000 & 1,000 \\
\hline 35 a 50 & ,333 & ,400 &, 007 & ,406 & 1,000 & 1,000 \\
\hline $\begin{array}{l}51 \text { a } \\
\text { más }\end{array}$ & ,319 & , 090 & ,000 & ,020 & 1,000 & 1,000 \\
\hline $\begin{array}{l}\text { Total } \\
\text { activo }\end{array}$ & 1,000 & & ,017 & 1,000 & & \\
\hline
\end{tabular}

Como se puede observar en la tabla $\mathrm{N}^{\circ} 7$, la puntuación para -ra tiene signo negativo y -se muestra el signo positivo. En cuanto a la edad, el valor para el grupo de jóvenes tiene signo negativo, mientras que el valor para el grupo de adultos y mayores, signo positivo. Las categorías que tienen igual signo se asocian. Lo anterior quiere decir que, en la muestra estudiada, estadísticamente, los jóvenes se asocian con un mayor uso de -ra, mientras que los hablantes de 
edades de 35 a más (adultos y mayores), aunque también evidencian un mayor uso de -ra que de -se, se asocian con esta última variante.

Esta información refuerza la idea de que la variante -se del imperfecto y pluscuamperfecto de subjuntivo se está perdiendo en el castellano limeño, como sucede en las otras variedades del español americano y de España. Estadísticamente, queda comprobada la predominancia del uso de -ra y la escasa aparición de -se en los más jóvenes. En los adultos y mayores, en cambio, aunque también se observa un uso mayoritario de -ra, aún hay una clara asociación de estos con una mayor frecuencia de aparición de -se.

De esta manera, parece ser que esta investigación de tiempo aparente es una prueba de un cambio en proceso: la pérdida de la variante -se. Aunque no es posible asegurar que el camino que sigan las formas del imperfecto y pluscuamperfecto de subjuntivo en el español limeño actual no sufra alteración, existe una alta probabilidad de que una forma como cantase ya no funcione como una opción válida ante cantara para ningún hablante de castellano de Lima en el futuro. Desde el siglo XVII, cuando -ra se empieza a usar en contextos donde antes solo se usaba -se, han pasado más de 400 años. En la actualidad, aún se registra que ambas formas siguen vigentes con una clara tendencia a la pérdida de -se. Estudios futuros serían necesarios para determinar cómo evoluciona la tendencia.

\section{Conclusiones}

Se justifica el estudio de -ra y -se como un caso de variación a partir del concepto de espacios de variabilidad. Este se constituye como una alternativa adecuada al de variable sociolingüística para el estudio de la variación no solo en el plano fonológico, sino más allá de este. Aunque en el presente trabajo no se han discutido los diferentes matices semánticos que involucran las formas analizadas $(-r a /-s e)$ —en la segunda sección, se mencionó que estos ya han sido comprobados-, el concepto de espacios de variabilidad es importante por ser más abarcador: permite estudiar casos de variación cuyas variantes no implican diferencias de significado y casos 
en los que sí se pueden encontrar tales diferencias en las formas alternantes.

Por otro lado, a partir del análisis de los datos, se comprueba el uso mayoritario de la variante -ra en detrimento de -se. En el total de casos analizados en este estudio, la forma -ra aparece el 79,6\% de las veces. Por su parte, -se alcanza un porcentaje de 20,4\% de aparición. En este sentido, se puede afirmar que la forma -se es la que menos se usa.

Respecto a la variable social edad, se comprueba que esta muestra asociación estadísticamente significativa con las formas de imperfecto y pluscuamperfecto de subjuntivo: mientras que -ra se asocia al grupo de hablantes jóvenes, -se está asociada a los grupos de adultos y mayores. Lo anterior refuerza la idea de que las formas verbales en -se se están perdiendo, ya que son los más jóvenes quienes menos las usan. Asimismo, se demuestra la importancia de los estudios en tiempo aparente para dar cuenta de un posible cambio en curso.

Además, se observó que, entre los adultos y mayores, son los adultos y no los mayores quienes usan más la variante -se. Lo anterior puede parecer contradictorio con el hecho de que sea esta forma la que se está perdiendo, pero se explica a partir del fenómeno de hipercorrección: la razón por la que los adultos presentan más usos de -se que los hablantes mayores reside en que esta es la forma considerada más prestigiosa o culta. Los entrevistados adultos (de 35 a 50 años), al desempeñarse todos en diferentes ámbitos profesionales, buscan usar formas que les permitan posicionarse como hablantes de la variedad más prestigiosa del castellano, lo que logran a través del uso de -se. Es interesante señalar que este resultado es bastante similar a aquel obtenido por Asratián (2007), al cual ya se hizo referencia en el segundo apartado de este trabajo.

Con el fin de confirmar los resultados de la presente investigación, es necesario recoger más datos de la variedad limeña. Asimismo, estos podrían compararse con los castellanos de otros espacios geográficos del Perú para conocer cuán extendido y generalizado se encuentra este fenómeno de variación en el país. Ya se 
vio, por ejemplo, que el patrón de uso mayoritario no se cumple para el español hablado en Chincha. Lo anterior sería muy interesante de estudiar al ir este último patrón retrasado con respecto a lo que se encuentra para la mayoría de variedades del castellano. Además, estudios futuros serán necesarios para saber cómo evoluciona esta aparente retención de la forma -se por hablantes adultos y su implicancia para detener el proceso de este posible cambio lingüístico.

\section{Referencias bibliográficas}

Alvar, Manuel y Bernard PotTier

1983 Morfología histórica del español. Madrid: Editorial Gredos.

AsRatián, Arucia

2007 "Variación -ra/-se en el español hablado en Caracas". Boletín de Lingüística. 19, 27, 5-41. Consultado: 9 de junio del 2014. <http://www.scielo.org.ve/scielo.php?pid=SO798$97092007000100001 \&$ script $=$ sci_arttext $>$

Blas Arroyo, José Luis y Margarita Porcar Miralles

1994 "Empleo de las formas -ra y -se en las comunidades de habla castellonenses. Aproximación sociolingüística”. Español actual. Revista de español vivo. 62, 73-98.

Caravedo, Rocío

1991 "Los espacios de variabilidad en fonología". Voz y Letra. Revista de Filología. II, 1, 17-38.

Caravedo, Rocío

2014 Percepción y variación lingüistica. Enfoque sociocognitivo. Madrid/Frankfurt: Iberoamericana/Vervuert.

Cuba, María

1996 El castellano hablado en Chincha. Lima: Escuela de Postgrado UNMSM.

Demello, George

1993 “-ra Vs. -se subjunctive: a new look at an old topic”. Hispania. $76,2,235-244$. 
Hernández, Roberto, Carlos Fernández y María Baptista

2010 Metodología de la investigación. Quinta edición. México: McGraw-Hill/Interamericana Editores, S.A.

Instituto Nacional de Estadística e Informática (INEI)

2007 Censos nacionales 2007: XI de Población y V de vivienda. Educación. Lima: Instituto Nacional de Estadística e Informática. Consultado: 8 de junio de 2016. <http://censos.inei.gob. pe/cpv2007/tabulados/\#>

Kempas, Ilpo

2011 "Sobre la variación en el marco de la libre elección entre cantara y cantase en el español peninsular”. Moenia. 17, 243-264. Consultado: 25 de mayo del 2014. <http://dspace.usc.es/ bitstream/10347/7332/1/7-Kempas.pdf $>$

Klee, Carol, Daniel Tight y Rocío Caravedo

2011 "Variation and Change in Peruvian Spanish Word Order: Language Contact and Dialect Contact in Lima". Southwest Journal of Linguistics. 30, 2, 5-31.

LABOv, William

1972 Sociolinguistics Patterns. Philadelphia: University of Pennsylvania Press.

LABOv, William

1978 "Where does the linguistic variable stop? A response to Beatriz Lavandera”. Working papers in Sociolinguistics. 44, 6-23.

LAMíQuiz, Vidal

1971 “Cantara y cantase”. Revista de Filología Española. LIV, 1-11.

LAVANDERA, Beatriz

1978 "Where Does the Sociolinguistic Variable Stop?". Language in Society. 7, 2, 171-182. <https://doi.org/10.1017/ S0047404500005510>

LAVANDERA, Beatriz

1979 "Análisis semántico de variación en tiempos verbales: oraciones condicionales del español”. Anuario de Letras. XVII, 113-135.

Moreno, Francisco

2009 Principios de sociolingüistica y sociología del lenguaje. Cuarta edición. Barcelona: Editorial Ariel S. A. 
Navarro, Manuel

1990 "La alternancia -ra/-se y -ra/-ría en el habla de Valencia (Venezuela)". Thesaurus. XLV, 2, 481-488. Consultado: 8 de junio de 2014. <http://cvc.cervantes.es/lengua/thesaurus/pdf/45/ TH_45_002_177_0.pdf>

Real Academia Española (RAE)

2009 Nueva gramática de la lengua española. Morfología y Sintaxis. Madrid: Espasa Libros.

Rojo, Guillermo y Victoria VÁsQueZ

2014 "Sobre las formas en -ra en el español de Galicia". En Perspectives in the study of spanish language variation. Papers in honor of carmen Silva-Corvalán. Eds., Andrés EnriqueArias, Manuel Gutiérrez, Alazne Landa y Francisco Ocampo. s/l.: Universidad Santiago de Compostela. Consultado: 8 de setiembre del 2016. <http:/gramatica.usc.es/ vvazq/pdf_ publ/Formas_en_ra_esp_de_Galicia.pdf>

Romaine, Suzanne

1981 "On the Problem of Syntactic Variation: A Reply to Beatriz Lavandera and William Labov". Sociolinguistic Working Paper. 82, 3-40.

Samamé, Anahís

2018a “¿Cantara o cantase?: un studio sociolingüístico del imperfecto y pluscuamperfecto del subjuntivo en hablantes limeños con grado de instrucción superior”. Tesis para optar el grado académico de Magíster en Lingüística. Pontificia Universidad Católica del Perú.

Samamé, Anahís

2018b "Superando el principio de equivalencia semántica: un caso de variación morfológica estudiado con el concepto de espacios de variabilidad". Lengua y Sociedad. 17, 2, 45-61.

SAnkoff, Gillian y Pierrette Thiabault

1980 "The alternation between tha auxiliaries avoir and être in Montréal Frech”. En The social life of language. Ed., Gillian Sankoff. Philadelphia: University of Philadelphia Press, 311345. 
Serrano, María José

1998 "Estudio sociolingüístico de una variante sintáctica: el fenómeno dequeísmo en el español canario". Hispania. 81, 2, 392-405. <https://doi.org/10.2307/345054>

Serrano, María José

2007 "Historia que ya es historia: evolución y actualidad del concepto y la metodología de la variación sintáctica”. Boletín de Lingüística. 19, 28, 102-127.

Tagliamonte, Sali

2006 Analysing Sociolinguistic Variation. Cambridge: Cambridge University Press.

Valeš, Miroslav

2006 "El prestigio desigual de las formas de imperfecto de subjuntivo cantara/cantase”. En Lexicografía y enseñanza de la lengua Española. Actas de las XI Jornadas sobre enseñanza de la lengua Española. Eds., Juan Antonio Moya Corral y Marcin Sosiński. Granada: Grupo de investigación "Estudios de Español Actual”, 303-312.

WeIner, Judith y William Labov

1983 "Constraints on the agentless passive". Journal of Linguistics. 19, 1, 29-58. <https://doi.org/10.1017/S0022226700007441>

Williams, Alan

1982 "The use of -ra and-seForms of the past subjunctive in Navarre". Hispania. 65, 1, 89-93. <https://doi.org/10.2307/341802>

Recepción: 13/11/2019 Aceptación: 30/11/2020 